\title{
The Clostridium perfringens enterotoxin gene is on a transposable element in type $A$ human food poisoning strains
}

\author{
Sigrid Brynestad, Bjørnar Synstad and Per Einar Granum
}

Department of

Pharmacology,

Microbiology and Food

Hygiene, Norwegian

College of Veterinary

Medicine, PO Box 8146,

Dep., N-0033 Oslo, Norway Author for correspondence: Per Einar Granum. Tel: +47229648 45. Fax: +4722964850.
e-mail: Per.E.Granum@veths.no

Keywords: Clostridium perfringens, enterotoxin, IS elements, cpe, transposon

\section{INTRODUCTION}

Clostridium perfringens is a Gram-positive, anaerobic, sporulating, ubiquitous bacterium which is important both in human and veterinary medicine. This species is divided into five subtypes (A-E) based on the major lethal toxins they produce. Type A can cause human food poisoning due to an enterotoxin produced during sporulation (Granum \& Stewart, 1993; Rood \& Cole, 1991). Other types of C. perfringens have been shown to carry the enterotoxin gene (Daube et al., 1994) but have not been shown to cause food poisoning. The enterotoxin gene $(c p e)$ can be chromosomally or plasmidbound (Cornillot et al., 1995), although in strains isolated from food poisoning outbreaks cpe is located on the chromosome. Observations that strains can suddenly lose or gain the ability to produce enterotoxin, along with the low prevalence of enterotoxin-positive strains from natural isolates (Van Damme-Jongsten et al., 1989), has led to speculation that cpe is carried on a transposable element. We have previously sequenced

The EMBLGenBank/DDBJ accession numbers for the sequences reported in this paper are X71844, Y09556, Y0557 and Y0558.
$4 \cdot 1 \mathrm{~kb}$ upstream of a chromosomally bound cpe and shown that it is associated with an IS200-like element (Brynestad et al., 1994), IS1469. The close association of this insertion element to cpe in other strains has been confirmed (Cornillot et al., 1995). The large episome to which plasmid-borne cpe has been mapped has been shown to harbour other toxin genes and IS elements (Daube et al., 1993).

Prokaryotic transposons have been detected in essentially all species of bacteria that have been examined. Transposons always carry a gene encoding a transposase which mediates transposition. They can also carry other genes, most notably antibiotic resistance genes, catabolic genes, viral genes and genes encoding virulence factors (reviewed in Craig, 1996). Most IS elements have terminal inverted repeats, which are recognition sites for the transposase, with IS200 being a notable exception (Lam \& Roth, 1986). There are certain 'hot spots' in the host DNA where insertion is more likely to occur. When the mobile element integrates into the target DNA it creates a duplication of a certain and characteristic number of base pairs from the host DNA (Galas \& Chandler 1989). Mobile elements are an important vehicle for movement of genetic material both within and between species. 


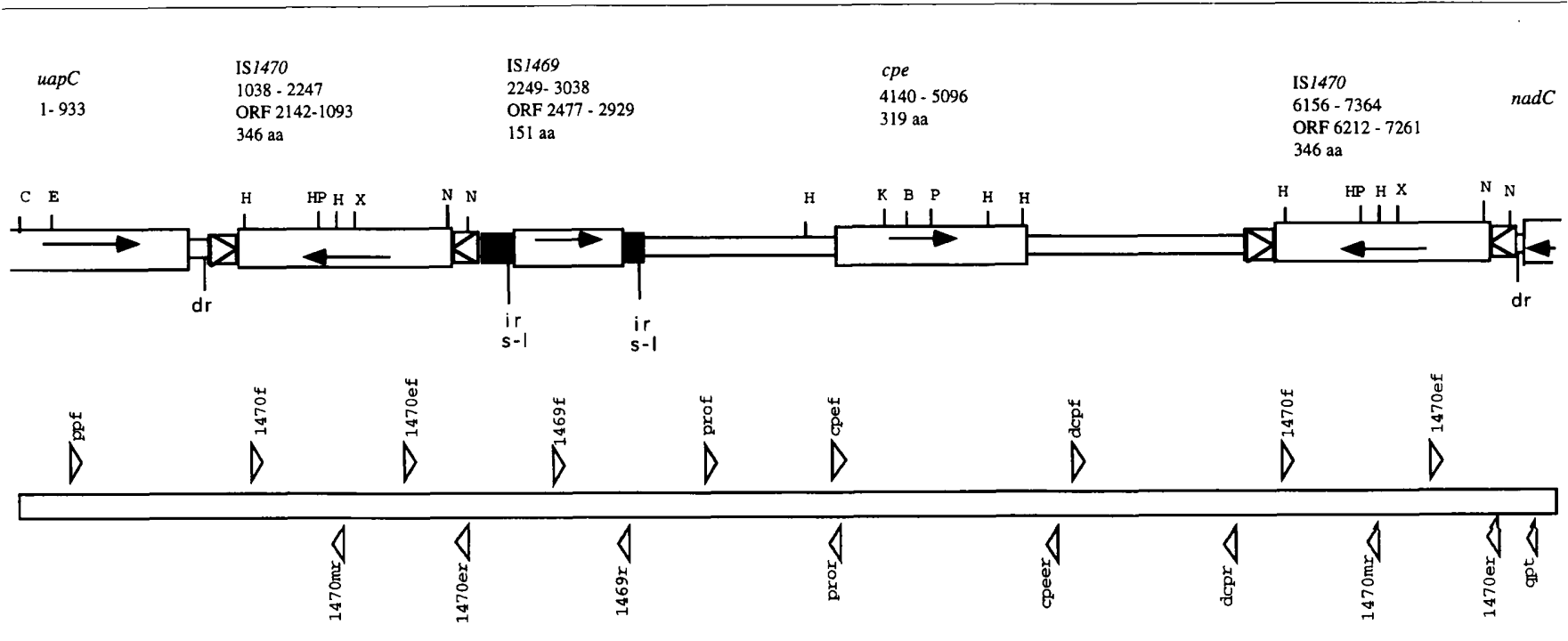

Fig. 1. Schematic representation of the entire sequenced region in NCTC 8239 . The white boxes show ORFs and the arrows indicate the coding strand and direction of transcription. Numbers under the IS elements indicate the total extent of the IS element including inverted repeats, while the length of the coding area of the putative transposase is given after ORF. Numbers under the other genes indicate the coding area. The uapC gene encodes purine permease, cpe encodes enterotoxin, and nadC encodes quinolinate phosphoribosyltransferase. Inverted repeats on each end of 151470 are indicated. The black boxes show the up- and downstream areas of IS1469, with the stem-loop structures (s-I) and inverted repeats (ir) indicated. The location of the direct repeat (CATAATATACAAAT) is shown (dr). Restriction enzyme sites are indicated: B, BgllI; C, Clal; E, EcoRI; H, HindIII; HP, Hpal; K, Kpnl; N, Ncol; P, Pstl; X, Xbal. The lower part of the figure shows the location of primers used in $P C R$ and sequencing reactions. The sequences of the primers are: 1470f, TCCTITCGGGATAAAACGC; 1470mr, TGAAAACCGTGAAGAATTTGG; 1470ef, GCTTAGTAGTACCACGGCGTATTTCG; 1470er, CACCATGGACTATCAAAATC; 1469f, ACATTTCTGGTGTATGGGA; 1469r, TGCTAGAGTTAAAAGTGTCC; prof, AATACATACGCAGGGTTAGAG; pror, ATTATAATTAACATCTCCTT; cpef, TCCAATGTGGTTCGAAAATG; cpeer, CATACTGTCTIITGTAAATT; depf, GTACATATCATTGGTGATAG; dcpr, GAATCTATCTAGGTAGCCAG; ppf, AAGCTATTGGAGAGACATCT; qpt, AAGTATTAGAGACATTAAGCTATG.

We were interested in defining a possible transposable unit that includes cpe to explain the sudden acquisition of enterotoxin-producing capabilities in food poisoning strains. We have previously mapped three open reading frames (ORFs) in the $3 \mathrm{~kb}$ region of the chromosome upstream of $c p e$ and were interested in finding the gene organization downstream of cpe. By cloning the $2 \mathrm{~kb}$ DNA $3^{\prime}$ to $c p e$ we have mapped the entire transposon as well as a downstream 'housekeeping' gene.

\section{METHODS}

Bacterial strains and growth conditions. NCTC 8239 and 2154 have been described by Brynestad et al. (1994) and are human food poisoning strains. Strain 44071-C05 was supplied by S. Cole (Institut Pasteur, Paris, France) and was isolated from goat intestine (Cornillot et al., 1995). A Norwegian hospital strain, 790-94, was obtained from the lungs of a patient who had died from complications after food poisoning. Strains were grown anaerobically in BHI medium (Difco). Epicurian Coli XL1 Blue cells or JM109 cells (Stratagene) were used in cloning experiments. Clones were grown in LB broth or on LB agar with $100 \mu \mathrm{g}$ ampicillin $\mathrm{ml}^{-1}$.

Isolation of DNA. Plasmids from Escherichia coli were isolated using QIA prep or QIAGEN plasmid Maxi kits, or as described by Sambrook et al. (1989). DNA was isolated from gels with a QIAex gel extraction kit. Chromosomal DNA from C. perfringens was isolated using ADVAMAX beads and the protocol supplied by the manufacturers for bacterial genomic DNA purification (Advanced Genetics Technologies).

PCR. PCR was carried out in an MJ Research Minicycler. The annealing temperature used was from $40^{\circ} \mathrm{C}$ to $55^{\circ} \mathrm{C}$, depending on the primers used (see Fig. 1 legend). The standard programme was: $92^{\circ} \mathrm{C}$ for $1 \mathrm{~min}$, annealing temperature for $1 \mathrm{~min}$ and $72^{\circ} \mathrm{C}$ for $1 \mathrm{~min}$ for 30 cycles. The low annealing temperatures are due to the high AT content of the $C$. perfringens genome. DYNAZyme DNA polymerase and buffer and $10 \mathrm{mM}$ dNTP from Finnzymes OY were used.

Hybridization. Various PCR products were used as probes. Blotting, the labelling of probes, and detection were performed as described in the ECL direct labelling and detection kit, using Hybond $-\mathrm{N}^{+}$as the nylon membrane for blotting (Amersham).

PCR screening of clones. Clones from transformations were screened using PCR. Colonies were picked and transferred to new LB agar plates containing ampicillin. Small amounts of cells were suspended in $100 \mu \mathrm{l}$ water, and microwaved for $3 \mathrm{~min}$. Two to five microlitres from this tube was used in a PCR reaction. Initial screenings were done with 10 colonies in each tube. The 10 bacteria from positive tubes were tested individually in a new PCR.

\section{Cloning of genes from different strains}

Strain NCTC 8239. The entire cpe gene with flanking areas was cloned into pUC18 as a $5 \cdot 1 \mathrm{~kb}$ Xbal fragment (see Fig. 1) using essentially the same strategy as by Czeczulin et al. (1993). The 
entire transposon could not be cloned, despite repeated attempts, and the rest of the downstream DNA was PCR cloned using the $3^{\prime}$ primer determined from 44071-C05 sequences described below.

Strain 44071-C05. ORF1 (purine permease) and IS1470 are on the chromosome, as in NCTC 8239, but with cpe and IS1469 located on a plasmid (Cornillot et al., 1995). A Southern blot showed that the DNA of interest was contained on an approximately $1 \mathrm{~kb}$ HindIII fragment. Fragments from the appropriate size range were purified from a gel, and ligated into pUC18; PCR was carried out on this ligation solution, using universal primers and a primer internal to IS1470 $(1470$ ef). The resulting PCR product was cloned and sequenced.

Strain 790-94. DNA was cloned using PCR products resulting from the use of qpt and $1470 \mathrm{f}$ primers (see Fig. 1).

Strain 2154. DNA was cloned using the product from qpt and ppf primers (see Fig. 1).

Cloning of PCR products. PCR products were cloned using the pMOSBlue T-vector kit (Amersham) following the manufacturer's instructions.

DNA sequencing. Sequencing was performed on a Perkin Elmer ABI Prism 377 automatic sequencer. ABI Prism Dye Primer or Dye Terminator Cycle Sequencing Ready Reaction kit were used following the manufacturer's instructions. Primers other than universal are listed in the legend to Fig. 1. All sequences from NCTC 8239 have been sequenced on both DNA strands. The cloned PCR products from the other strains were, in general, sequenced from both strands. In the cases where there was not complete overlap, the same strand was sequenced in separate reactions.

\section{RESULTS AND DISCUSSION}

\section{Characterization of ORFs upstream of cpe}

We had previously sequenced $4.1 \mathrm{~kb} 5^{\prime}$ to the enterotoxin gene (cpe) in C. perfringens NCTC 8239 (Brynestad et al., 1994). New entries in the databases, along with corrections in the sequence, gave identities to the two unidentified ORFs and a better total homology to IS200 for ORF3. ORF1 has high homology to the purine permease gene uapC in Aspergillus nidulans (Diallinas et al., 1995). This is a housekeeping gene, and has a stable position on the chromosome of the strains that have been examined. ORF2 has been registered, according to convention, as IS1470 (Lederberg, 1987). ORF3 has been registered as IS1469. An overview of the region, including the $3^{\prime}$ DNA, is given in Fig. 1.

\section{Analysis of IS1469}

IS1469 is an IS200-like element with a $453 \mathrm{bp} \mathrm{ORF,}$ encoding a 151 aa protein, corresponding to other known IS200-like elements with greater than $60 \%$ homology, both at amino acid and DNA levels, in the coding area. Most of the DNA differences are in the third 'wobble' position, with shifts to $\mathrm{T}$ or $\mathrm{A}$, reflecting the high AT content of C. perfringens. This particular group of IS elements, which was first thought to be specific to Salmonella spp., appears to be highly conserved in a number of bacterial species (Fig. 2). The high degree of identity of IS1469 to IS200 elements found in
Salmonella typhimurium, Yersinia pestis, E. coli and Vibrio cholerae was rather surprising considering the relatively large phylogenetic distance between these bacteria and $C$. perfringens, and suggests horizontal transfer of IS1469. As both C. perfringens and E. coli are part of our normal intestinal flora, they should have had ample opportunity for exchanging genetic material, through transfer of naked DNA, plasmids or through phages. Several lines of evidence point to a phage origin: the acquisition pattern of $c p e$, the fact that the enterotoxin is a superantigen (Bowness et al., 1992), and the absence of 6-base restriction endonuclease sites in the DNA surrounding the cpe.

The entire IS200 element from Salmonella spp. is $709 \mathrm{bp}$ (153 aa transposase) (Lam \& Roth, 1983, 1986; O’Reilly et al., 1990). IS1469 appears to be somewhat larger (789 bp) based on sequencing of the IS1469 from strain 44071-C05. The elements in the IS200 family are unusual in that they do not contain inverted repeats on each end of the IS element. A comparison in four species of bacteria of regions up- and downstream to the ORF showed two interesting features. E. coli, Y. pestis and S. typhimurium all have $>90 \%$ homology in the upstream region, which contains a potential stem-loop structure. In C. perfringens, there is minimal homology to the other bacteria in the upstream region, but it does contain a potential stem-loop structure (Figs 1 and 2), which is positioned closer to the ORF than in the other species. Downstream to the ORF there are two blocks of highly conserved DNA regions in all four species, one of which potentially forms a stem-loop structure and defines the end of the IS element.

We have not yet performed studies on expression or transposition on IS1469. Studies performed on the IS200 from S. typhimurium (Haack, 1995) indicated that the element transposes through a replicative cointegrative RecA-dependent mechanism. The $\operatorname{rec} A$ gene from $C$. perfringens has been cloned (Johnston et al., 1997) and complements a recA mutant in $E$. coli, so theoretically there should be no hindrance to active transposition. The transposition studies also showed that expression was undetectable unless the upstream stem-loop structure was removed. The stem-loop upstream of IS1469 is closer to the start codon of the transposase than in the other bacteria (Fig. 2), but there is a potential promoter immediately upstream which could be used after the removal of the stem-loop structure. The fact that there is only a single copy of IS1469 suggests that this is not a very active transposon and probably does not play an important role in the types of genome rearrangements described by Haack \& Roth (1995). IS1469 is found $1 \mathrm{~kb}$ upstream from almost all cpe genes, and in all type A strains tested, on both plasmids and chromosomes.

\section{Analysis of the 5' copy of IS1470}

IS1470 has been shown, using FASTA, to have $36 \%$ amino acid homology with IS1161 from Streptococcus salivarius, and $30 \%$ homology with IS 30 from E. coli, and it is considered to be part of the IS30 family, which 


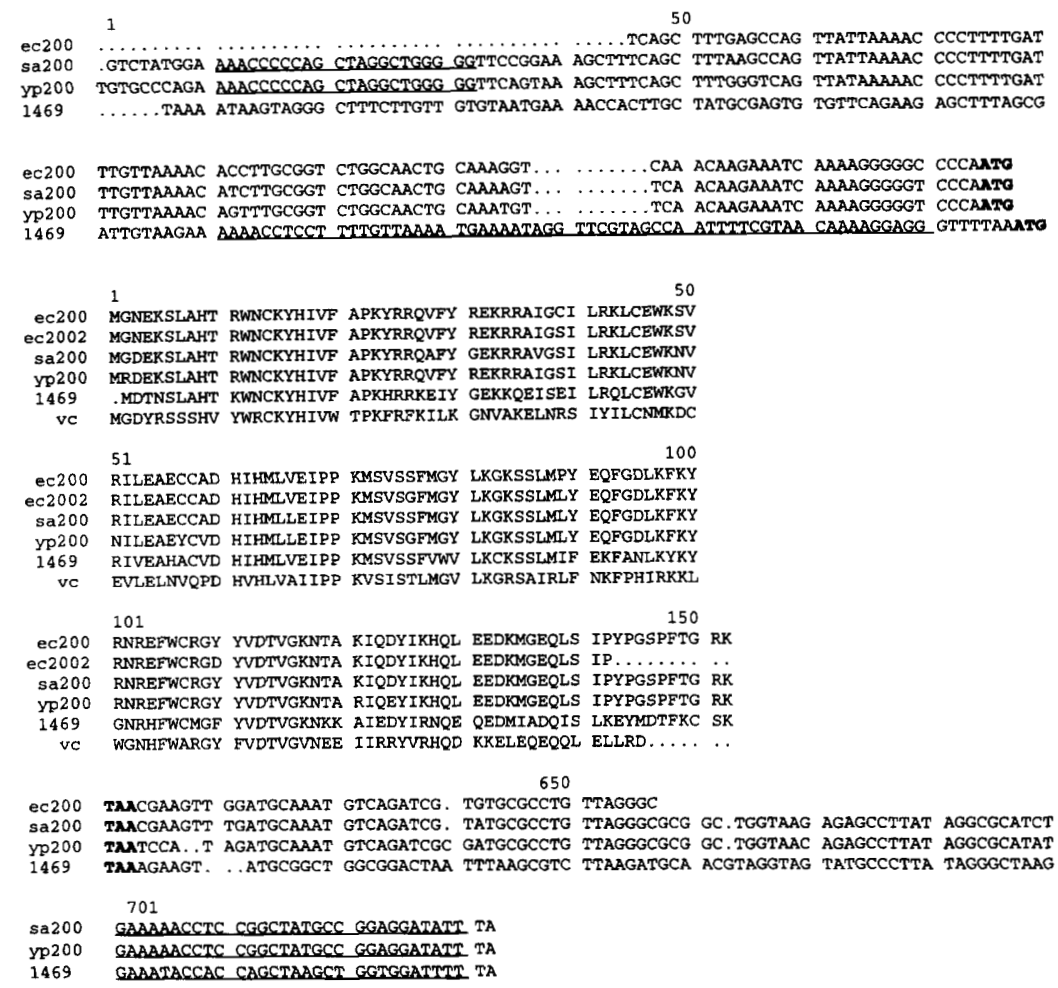

Fig. 2. PILEUP figure of various IS200 sequences. Up- and downstream DNA and amino acids of the transposase are shown. The DNA is shown for $E$. coli (ec200), S. typhimurium (sa200), Y. pestis (yp200) and $C$. perfringens (1469). The amino acid sequence is also shown for $E$. coli (ec2002) and $V$. cholerae (vc). Start and stop codons are shown in bold type. The potential stem-loop structures are underlined. presently has 19 members (J. Mahillon \& M. Chandler, unpublished). The 'DDE signature' (Kulkosky et al., 1992; Rezsöhazy et al., 1993) and other conserved amino acids in the integrase domain are present (Fig. 3). There are inverted repeats of $26 \mathrm{bp}$, with four mismatches, flanking this element.

IS1470, a member of the IS30 family of transposases, is found in multiple copies (at least eight) in NCTC 8239, but the number per genome can vary from 0 to more than 10 in different strains of C. perfringens (Brynestad et al., 1994; Cornillot et al., 1995). It is tempting to postulate that IS1470 transposes by the same mechanism as the well-studied IS30, but we have no experimental evidence yet that shows the mode of transposition. What we have noticed is that the configuration which is very stable in the $C$. perfringens chromosome becomes very unstable when placed in a cloning vector in E. coli. We have never successfully cloned DNA with both copies of IS1470. Those who have isolated such a clone found that the plasmid had always undergone rearrangements and was present in high copy numbers, despite being cloned in a low copy number E. coli vector (G. Stewart, personal communication).

Studies on the transposition mechanism of IS30 show that the formation of a tandem repeat (IS30) ${ }_{2}$ with an unstable circular intermediate plays an important role in transposition, and possibly gene rearrangement (Olasz et al., 1993). Since we see the same type of organization of IS1470 on the genome of C. perfringens we can speculate that the same type of reactions occur in this bacterium. The existence of circular transposons in $C$. perfringens has been shown (Bannam et al., 1995), and these were shown to be able to transpose into the E. coli genome.

\section{Cloning of DNA $3^{\prime}$ to cpe}

A $5 \cdot 1 \mathrm{~kb} \mathrm{XbaI}$ fragment containing $c p e$, which includes $2.4 \mathrm{~kb}$ upstream and $1.7 \mathrm{~kb}$ downstream of $c p e$, was cloned and the downstream DNA was sequenced. Analysis of the sequence showed that there was another copy of IS1470, 1.1 kb downstream of $c p e$. The intervening $1.1 \mathrm{~kb}$ DNA was, as the DNA upstream of $c p e$, devoid of 6-base cutting restriction enzymes and ORFs, and showed no significant similarity with other sequences in the databases.

\section{Cloning of a partial putative nadC (quinolinate phosphoribosyltransferase) gene from strain 44071- C05}

Since $X b a \mathrm{I}$ cuts internally in the transposon gene, the sequence of the entire gene was not obtained. We reasoned that genes linked to ORF1 in strains without or with episomally located cpe would be located downstream of cpe in NCTC 8239. Strain 44071-C05 was, therefore, utilized (see Methods). Sequencing of the PCR product showed two putative partial ORFs $3^{\prime}$ to IS1470, one on each strand in an overlapping area. In BLAST and FASTA searches, one of these putative ORFs with a length of 77 aa gave $48 \%$ homology with the $3^{\prime}$ end of the nadC gene from E. coli, and had homology with nadC genes from other species. The other ORF had no homology to known genes. The nadC gene encodes quinolinate phosphoribosyltransferase, which is part of the pyridine 


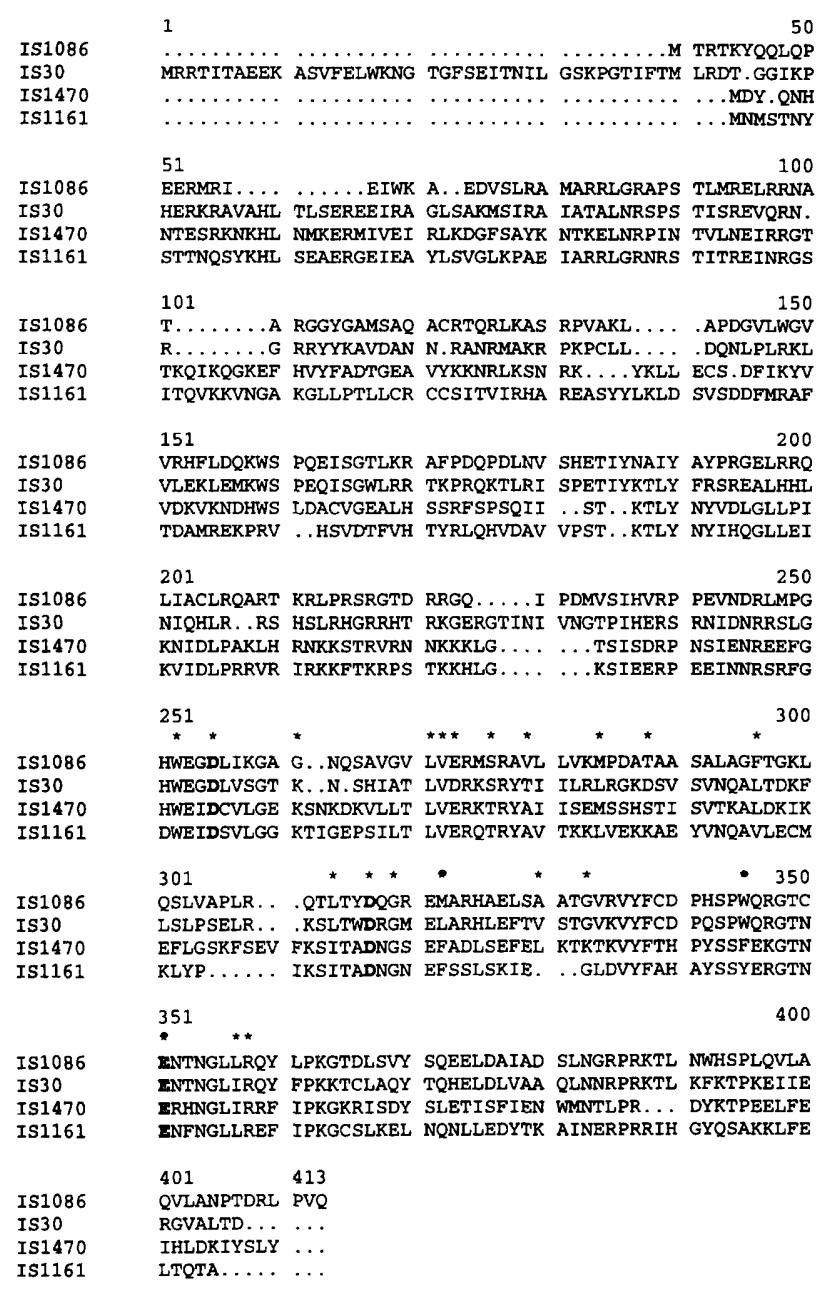

Fig. 3. PILEUP figure of IS1470 and three of the transposases in the IS30 family. IS1086 was isolated from Alcaligenes eutrophus, IS30 from E. coli and IS1161 from Strep. salivarius. As in the whole IS 30 family, the $5^{\prime}$ recognition end is less conserved than the $3^{\prime}$ integrase domain. The amino acids which are highly conserved in the family are indicated by an asterisk (Rezsobhazy et al., 1993). The essential DDE binding motif amino acids are shown in bold type.

nucleotide synthesis pathway. PCR results showed that ' $n a d C$ ' is proximal to IS1470 in NCTC 8239, and is proximal to the uapC gene in enterotoxin-negative strains, and in strains where cpe is plasmid-bound.

\section{Cloning of the downstream copy of IS1470 in NCTC 8239}

The sequence of the downstream copy of IS1470 was completed using $1470 \mathrm{f}$ and qpt primers on DNA from NCTC 8239 . It has $98 \%$ identity on the DNA level, and a $96 \%$ identity (97\% similarity) with 12 aa changes as compared to the upstream copy. The putative inverted repeats were conserved on both ends of the downstream copy. There is $26 \mathrm{bp}$ between the putative inverted repeats and the stop codon in nadC, which is 204 bp less than was found in 44071-C05. A 14 bp directly repeated region was found directly before the initial inverted repeats of the first copy of IS1470 and immediately after the inverted repeats on the $3^{\prime}$ end of the second copy (Fig. 1).

\section{Comparison of DNA between uapC or IS1470 and nadC in various strains}

The region between $u a p C$ and nadC should contain the target sequence for insertion. As the results indicated that NCTC 8239 had lost 204 bp compared to 44071C05, PCR products from two other strains were sequenced (see Methods). In strain 2154, which has lost cpe, the $317 \mathrm{bp}$ PCR product using ppf and qpt primers was homologous to the first $87 \mathrm{bp}$ of the uapC-IS1470 sequence in NCTC 8239 . The 14 bp direct repeat (see above) was found once, and the rest of the sequence was identical to that in 44071-C05, which indicates that IS1470 duplicated $14 \mathrm{bp}$ from the host genome upon insertion. The 1470 ef-qpt PCR product from strain 790 94 was identical to $44071-\mathrm{C} 05$ (Fig. 4). As IS1470 is part of the IS30 family, the consensus sequence for insertion of IS30 was searched for, but not found (Stalder et al., 1987). PCR of the area between uapC and nadC in enterotoxin-negative strains showed that this region is somewhat variable in size (not shown). A FASTA search of the DNA between nadC and IS1470 showed that there was $88 \%$ identity over $97 \mathrm{bp}$ to the DNA found downstream of the gene $s s p C 1$ encoding the acid-soluble spore protein from $C$. perfringens. The loss of this region has no effect on cpe transcription, as judged from the amounts of enterotoxin produced during sporulation (unpublished observations). It is possible that recombination of homologous DNA has deleted the region.

\section{Configuration of cpe}

NCTC 8239 is a type A strain originally from the UK which has been used since 1952 by laboratories all over the globe. Strain 790-94 was isolated recently from a patient in Norway. Only a $204 \mathrm{bp}$ difference was observed between these two strains. The NCTC strain has 'lost' these bases between IS1470 and nadC (Fig. 4). There are two regions of about $1 \mathrm{~kb}$ flanking cpe on this transposon which have no ORFs, and almost no restriction enzyme sites. Only the promoter area $5^{\prime}$ of $c p e$ would seem to have any vital function. Yet this DNA is conserved. Such large chromosomal DNA domains without ORFs are atypical. A look at the databases of the genomes of Mycoplasma (Fraser, 1995) and Haemophilus (Fleischmann et al., 1995), which are entirely sequenced, and E. coli, much of which is sequenced, shows that the maximum length of DNA without a coding function is about $1 \mathrm{~kb}$. Such non-coding regions are, however, always located on one side of an operon, and do not flank a single gene. The same cpe-flanking DNA is also found in the strain 44071-C05, which has cpe on a plasmid. On this plasmid, cpe is linked to IS1469 but not coupled to IS1470. An explanation for the conservation of this flanking DNA can be that this region contains no sequences which would give a basis for homologous recombination and excision. In ad- 


uapC

dition, several pyrimidine tracts are found in this DNA, and these are known to give a rigid structure (Calladine \& Drew, 1992) which could impair recombination.

The physical map of the chromosome of C. perfringens has almost 100 markers. The enterotoxin gene has been shown to be carried on a $5 \mathrm{~kb} \mathrm{NruI} \mathrm{fragment} \mathrm{when}$ found on the chromosome (Canard et al., 1992; Cornillot et al., 1995). The larger $370 \mathrm{~kb}$ I-CeuI fragment which encompasses the gene shows signs of rearrangement involving both insertion and deletion in strains where the enterotoxin is on the chromosome. In enterotoxin-negative strains, and strains where cpe is plasmid-borne, the arrangement of this region is constant. The majority of enterotoxin-positive strains carry the gene on a plasmid, and almost all of these strains have IS1469 upstream from cpe, but do not have IS1470 on the plasmid. Plasmid-borne copies of cpe are often found in association with another IS element, IS1151 (Daube et al., 1993), and the episome also carries other toxin genes. Most of these strains with episomally located cpe are of animal origin, and enterotoxin production is still regulated by sporulation. Human food poisoning strains have cpe on the chromosome in the transposon described here. Since the IS elements associated with, and the genetic localization of, cpe seem to vary from human food poisoning to animal strains, it is interesting to speculate when and why these changes in genetic structure occur. The major difference in the environments between the strains isolated from animals and those from human food poisoning cases is the exposure to heat treatment in food for the human strains. Strains which are enterotoxin-positive and are passed repeatedly on medium without heat shocking can lose the enterotoxin gene (P. E. Granum, unpublished observations). How heat treatment affects the movement and expression of $c p e$ is one aspect of the production of enterotoxin which should be examined further.

\section{ACKNOWLEDGEMENTS}

This work has been supported by the Research Council of Norway, grant 105064/110.

\section{REFERENCES}

Bannam, T. L., Crellin, P. K. \& Rood, J. I. (1995). Molecular genetics of the chloramphenicol-resistance transposon $\mathrm{Tn} 4451$ from Clostridium perfringens: the $\mathrm{TnpX}$ site-specific recombinase excises a circular transposon molecule. Mol Microbiol 16, 535-551.

Bowness, P., Moss, P., Tranter, H., Bell, J. I. \& McMichael, A. J. (1992). Clostridium perfringens enterotoxin is a superantigen reactive with human $T$-cell receptors $v$-beta-6.9 and receptor v-beta-22. J Exp Med 176, 893-896.

Brynestad, S., Iwanejko, L. A., Stewart, G. \& Granum, P. E. (1994). A complex array of $\mathrm{Hpr}$ consensus DNA recognition sequences proximal to the enterotoxin gene in Clostridium perfringens type A. Microbiology 140, 97-104.

Calladine, C. R. \& Drew, H. R. (1992). Different kinds of double helix. In Understanding DNA: The Molecule and How it Works, pp. 41-65. London: Academic Press.

Canard, B., Saint-Joanis, B. \& Cole, S. T. (1992). Genomic diversity and organization of virulence genes in the pathogenic anaerobe Clostridium perfringens. Mol Microbiol 6, 1421-1429.

Cornillot, E., Saint-Joanis, B., Daube, G., Katayama, S., Granum, P. E., Canard, B. \& Cole, S. T. (1995). The enterotoxin gene (cpe) of Clostridium perfringens can be chromosomal or plasmid-borne. Mol Microbiol 15, 639-647.

Craig, N. L. (1996). Transposition. In Escherichia coli and Salmonella: Cellular and Molecular Biology, pp. 2339-2362. Edited by F. C. Neidhardt and others. Washington, DC: American Society for Microbiology.

Czeczulin, J. R., Hanna, P. C. \& McClane, B. A. (1993). Cloning, nucleotide sequencing, and expression of the Clostridium perfringens enterotoxin gene in Escherichia coli. Infect Immun 61, 3429-3439.

Daube, G., Simon, P. \& Kaeckenbeeck, A. (1993). IS1151, an IS-like element of Clostridium perfringens. Nucleic Acids Res 21, 352.

Daube, G., China, B., Simon, P., Hvala, K. \& Mainil, J. (1994). Typing of Clostridium perfringens by in-vitro amplification of toxin genes. J Appl Bacteriol 77, 650-655.

Diallinas, G., Gorfinkiel, L., Arst, H. N., Cecchetto, G. \& Scazzocchio, C. (1995). Genetic and molecular characterisation of a gene encoding a wide specificity purine permease of Aspergillus nidulans reveals a novel family of transporters conserved in prokaryotes and eukaryotes. J Biol Chem 270, 8610-8622.

Fleischmann, R. D., Adams, M. D., White, O. \& 37 other authors (1995). Whole-genome random sequencing and assembly of Haemophilus influenzae Rd. Science 269, 469-512. 
Fraser, C. M. (1995). The minimal gene complement of Mycoplasma genitalium. Science 270, 397-403.

Galas, D. J. \& Chandler, M. (1989). Bacterial insertion sequences. In Mobile DNA, pp. 109-162. Edited by D. E. Berg \& M. M. Howe. Washington, DC: American Society for Microbiology.

Granum, P. E. \& Stewart, G. S. A. B. (1993). Molecular biology of Clostridium perfringens enterotoxin. In Genetics and Molecular Biology of Anaerobic Bacteria, pp. 235-247. Edited by M. Sebald. New York: Springer.

Haack, K. R. (1995). The activity of IS200 in Salmonella typhimurium. PhD thesis, The University of Utah.

Haack, K. R. \& Roth, J.R. (1995). Recombination between chromosomal IS200 elements supports frequent duplication formation in Salmonella typhimurium. Genetics 141, 1245-1252.

Johnston, J. L., Sloan, J., Fyfe, J. A. M., Davies, J. K. \& Rood, J. I. (1997). The recA gene from Clostridium perfringens is induced by methyl methanesulphonate and contains an upstream Cheo box. Microbiology 143, 885-890.

Kulkosky, J., Jones, K. S., Katz, R. A., Mack, J. \& Skalka, A. M. (1992). Residues critical for retroviral integrative recombination in a region that is highly conserved among retroviral retrotransposon integrases and bacterial insertion-sequence transposases. Mol Cell Biol 12, 2331-2338.

Lam, S. \& Roth, J. R. (1983). IS200 - a Salmonella-specific insertion-sequence. Cell 34, 951-960.

Lam, S. \& Roth, J. R. (1986). Structural and functional studies of insertion element IS200. J Mol Biol 187, 157-167.
Lederberg, E. M. (1987). Plasmid Reference Center Registry of transposon ( $\mathrm{Tn}$ ) and insertion sequence (IS) allocations through December 1986. Gene 51, 115-118.

Olasz, F., Stalder, R. \& Arber, W. (1993). Formation of the tandem repeat $(\mathrm{IS} 30)_{2}$ and its role in IS30-mediated transpositional DNA rearrangements. Mol Gen Genet 239, 177-187.

O'Reilly, C., Black, G. W., Laffey, R. \& McConnell, D. J. (1990). Molecular analysis of an IS200 insertion in the $g p t$ gene of Salmonella typhimurium lt2. J Bacteriol 172, 6599-6601.

Rezsőhazy, R., Hallet, B., Delcour, J. \& Mahillon, J. (1993). The IS4 family of insertion sequences: evidence for a conserved transposase motif. Mol Microbiol 9, 1283-1295.

Rood, J. I. \& Cole, S. T. (1991). Molecular genetics and pathogenesis of Clostridium perfringens. Microbiol Rev 55, 621-648.

Sambrook, J., Fritsch, E. F. \& Maniatis, T. (1989). Molecular Cloning: a Laboratory Manual, 2nd edn. Cold Spring Harbor, NY: Cold Spring Harbor Laboratory.

Stalder, R., Konig, P. \& Arber, W. (1987). The target specificity of the prokaryotic mobile genetic element IS30. Experientia 43, 636.

Van Damme-Jongsten, M., Wernars, K. \& Notermans, S. (1989). Cloning and sequencing of the Clostridium perfringens enterotoxin gene. Antonie Leeuwenhoek J Microbiol 56, 181-190.

Received 4 November 1996; revised 24 February 1997; accepted 4 March 1997. 